\title{
3D Manufacturing Feature Based Integrated Information Model and its Modeling System Development Based on XML and NX Platform
}

\author{
Yan $\mathrm{Cao}^{1,2, \text { a }}$, Zhongcan $\mathrm{Lin}^{2}$, Yonglou Zhao ${ }^{2}$, Changzhong Yuan ${ }^{2}$ and Yunfeng \\ Chen $^{2}$ \\ ${ }^{1}$ Xi'an Technological University, Xi'an, Shaanxi, China \\ ${ }^{2}$ Shaanxi Space Pump \& Valve Science and Technology Co.,Ltd, Xi'an, Shaanxi, China \\ a jantonyz@163.com
}

\begin{abstract}
Keywords: CAD/CAPP, 3D Geometric model, Feature model, Integrated information model, NX, $\mathrm{XML}$, System development.
\end{abstract}

\begin{abstract}
With the development of CAD/CAM technology, the key to CAD/CAM integration consists in constructing a common product model. In the paper, shaft parts are taken as research objects. Interactive feature-based modeling method is adopted to avoid complex algorithms for feature recognition. The functional framework of the system developed is put forward. 3D geometric model is used as the core to integrate all manufacturing feature data of a part. All related manufacturing data are organized in a feature model and stored in a XML file. The 3D geometric model and corresponding feature XML file constitute an integrated information model for the part. In the modeling process, information consistency examination is carried out to check the feature model and judge whether a feature in the feature model should be modified. The system has already been used to realize a 3D CAD/CAPP.
\end{abstract}

\section{Introduction}

With the development of CAD/CAM technology [1], the key to CAD/CAM integration consists in constructing a common product model. The core of modern CAD systems is solid modeling. There exist two main solid representations.. The one is Boundary Representation (B-rep). The other is Constructive Solid Geometry (CSG).

The above two methods cannot represent the features of a part. In order to describe non-geometric information, features are used to complement a solid model [2]. A feature is a set of data concerned with a part or a product, including design data, manufacturing data, inspection data, managerial data, etc that are different from each other and represent different contents in different formats at different levels [3-5]. There are many classification ways, features generally can be divided into form feature, material feature, precision feature, technological feature, managerial feature, etc.

In the paper, based on the 3D geometric model constructed in NX, all manufacturing feature data are combined with the entities of the 3D geometric model. A XML file is used to represent the manufacturing feature data and their relations with geometric entities. Therefore, the 3D geometric model is converted into an integrated information model by combining the 3D geometric model with the XML file. Based on the integrated information model, a system is developed on NX platform. Herein, UIstyler, UG/Open MenuScript and UG/Open API are utilized to develop the system in Visual C++ 6.0 programming environment. In the paper, shaft parts are taken as research objects.

\section{Functional Framework of Manufacturing Feature Modeling System}

In the paper, interactive feature-based modeling method is adopted to avoid complex algorithms for feature recognition. It is different from feature-based modeling and can facilitate the addition of machining information to make the feature model satisfy the practical needs better. The functional framework of the system developed is shown in Fig.1. 


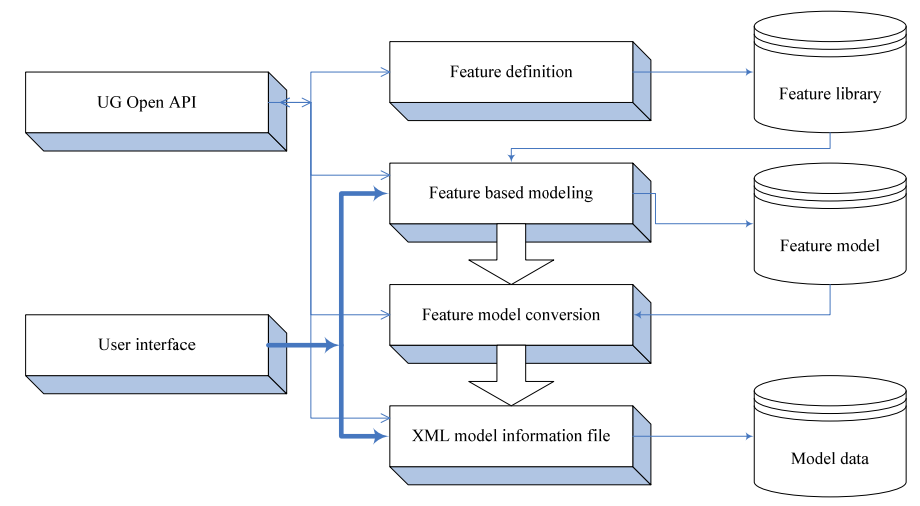

Fig.1 Functional framework of manufacturing feature modeling system

\section{Integrated Information Model}

A feature model of a part contains its geometry, dimension, manufacturig data and so on that are the key to information integaration between CAD, CAPP and CAM.

On the one side, the feature model should represent all feature data of the part. On the other side, the feature model should have a rationable structure to facilitate the organization and use of fearture data. The feature model structure adopted in the paper is shown in Fig.2. All data are divided into four levels as follows:

$>\quad$ The first level is part level. It describes part name, type, etc.

$>$ The second level is feature level. It describes all the features that the part includes and the relations among them. They mainly include parent feature ID, coupling relation, orientation relation, and feature elements that constitute the feature.

$>$ The third level is feature element level. It describes feature element data. Different feature possesses different feature elements, such as dimension, precision, orientation, form tolerance, position tolerance, surface quality, etc.

$>$ The forurth level is element attribute level. It describes feature element value, such as, precision grade, basic deviation symbol, form and position tolerance type, roughness, etc.

It is better that the feature model structure can direct reflect the part structure. In order to achieve the objective, a tree structure is used to represent the feature model. The corresponding XML document tree is shown in Fig.3.

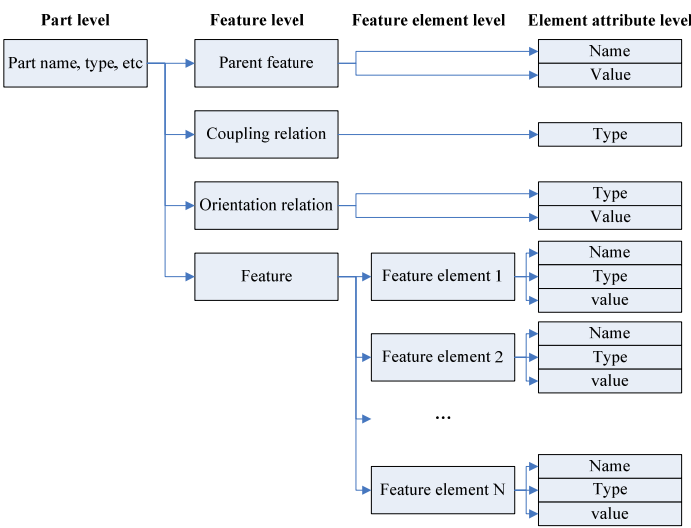

Fig.2 Feature model structure

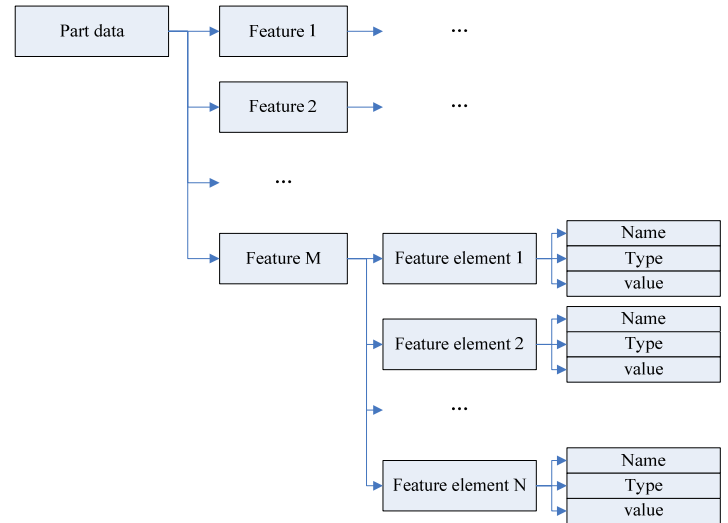

Fig.3 XML document tree

In the paper, the 3D geometric model is used as the core to integrate all manufacturing feature data of the part. All related manufacturing data are organized in the feature model and stored in a XML file. The 3D geometric model and corresponding feature XML file constitute an integrated information model for the part, as shown in Fig.4. In the feature model, the manufacturing data are attached to the related geometric entities. 


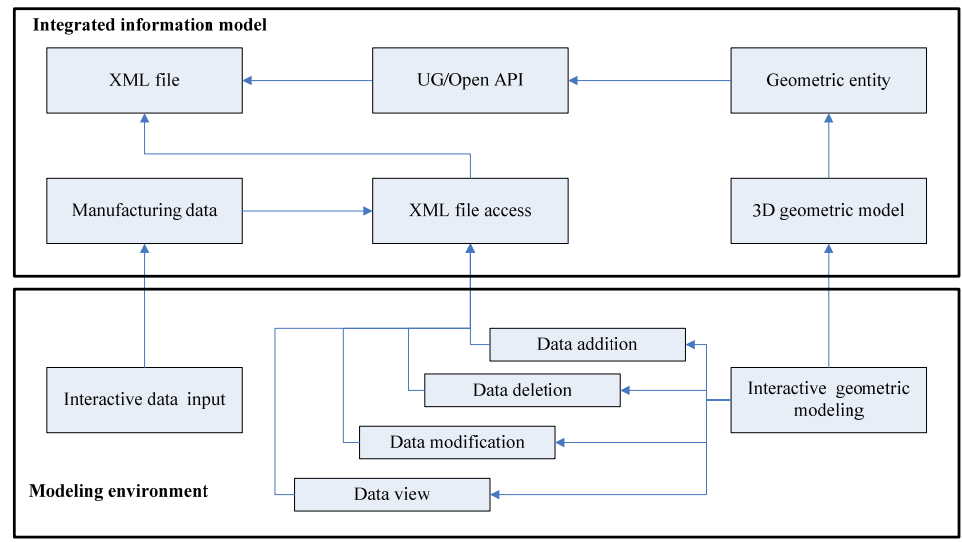

Fig.4 Integrated information model and its modeling environment

\section{Feature Model Data Input and Modification}

Based on the constructed model, feature model modification can be carried out to add, delete and modify feature data. In the modification process, information consistency examination is carried out to check the feature model and judge whether a feature in the feature model should be modified. If so, the feature is modified to assure the consistency of the integrated information model. model. After selecting feature type and inputing feature parameters of a feature, the procee returns to model the next feature. Finally, if the model is completed and passes through information consistency examination, the feature XML file is output that contains all the manufacturing feature data.

Part Data Input and View. Data input includes part data and feature data that are stored in the XML format file and can be queried. For example, part data input dialog box is shown in Fig.5 and part data view dialog box is shown in Fig.6.

\begin{tabular}{|c|c|c|}
\hline D零件信 & & $\underline{x}$ \\
\hline 零件名称 & & 轴 \\
\hline 设计者 & & 麽某 \\
\hline 毛坯种类 & & 铸件 \\
\hline 编号 & & 201-01 \\
\hline 材料 & & 铸铁 \\
\hline 热处理 & & 厎温淬火 \\
\hline 确定 & 应用 & 取消 \\
\hline
\end{tabular}

Fig.5 Part data input

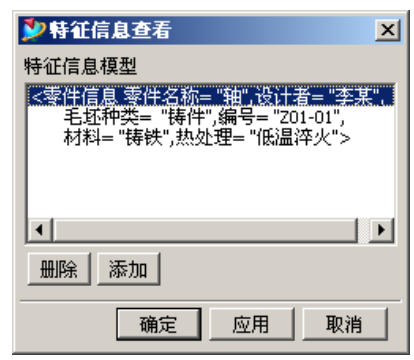

Fig.6 Part data view

Feature Data Input and View. According to the stepped feature definition of a shaft, the feature data addition of a stepped shaft is shown in Fig.7. They include basic dimensions, form and position precisions, and surface quality. Basic dimesions include diameter, length, precision grades and basic deviation symbols. Form and position tolerances include tolerance type, value, reference and principle. Surface quality includes axial region roughness, end face roughness and surface treatment. The feature data that have been input can be viewed, as shown in Fig.8. The feature data are displayed in the dialog box in XML format. If needed, the data can also be added or deleted in the dialog box.

\section{Application}

The modules developed are close integrated with NX functions to form an unified user interface. In the system development, in terms of UG development interface, features are defined by programming to form feature library. Then, feature-based modeling algorithm is programmed to construct the feature model. And then, the feature model in converted into the feature XML file. The system has already been used to realize a 3D CAD/CAPP. 


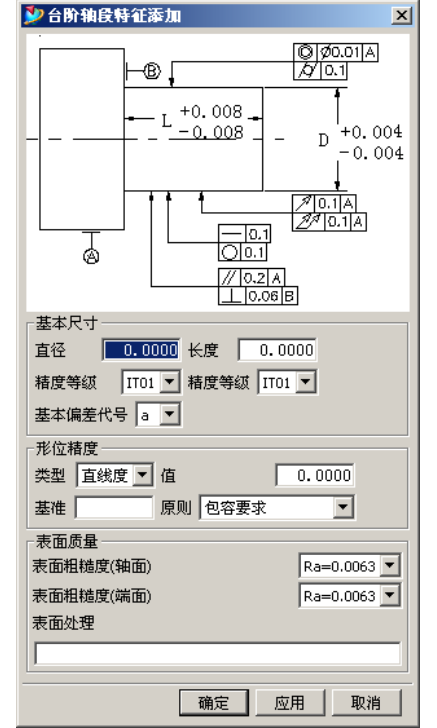

Fig.7 Stepped feature addition

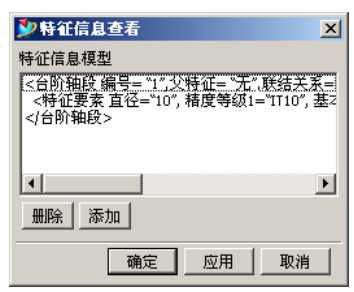

Fig.8 Feature data view

\section{Conclusions}

Feature modeling technology is the requirement of Advanced Manufacturing Technology (AMT) development. It is one of the key technologies of integrated CAD/CAPP/CAM system to build a shared part model. In the paper, based on the 3D geometric model constructed in NX, all manufacturing feature data are combined with the entities of the 3D geometric model. Therefore, the 3D geometric model is converted into the integrated information model by combining the 3D geometric model with the feature XML file. The system is developed on NX platform using UIstyler, UG/Open MenuScript and UG/Open API in Visual C++ 6.0 programming environment. In the paper, shaft parts are taken as research objects. The system has been used to realize a 3D CAD/CAPP.

\section{Acknowledgements}

The paper is supported by Natural Science Foundation of China (50405029), National 863 Project (2006AA04Z142), Shaanxi Major Subject Construction Project, Special Scientific Research Project of Shaanxi Education Office (06JK274) and President Scientific Research Fund of Xi'an Technological University (XGYXJJ0508).

\section{References}

[1] J. G. Cherng, X. Y. Shao, Y. B. Chen and P. R. Sfero: Computers \& Industrial Engineering Vol. 34 (1998), p. 515-530

[2] W. D. Li, S. K. Ong and A. Y. C. Nee: Computer-Aided Design Vol. 34 (2002), p. 849-868

[3] Lee Jae Yeo and Kwangsoo Kim: Computer-Aided Design Vol. 28 (1996), p. 831-841

[4] D. C. Anderson and T. C. Chang: Computers \& Graphics Vol. 14 (1990), p. 225 235

[5] Salomons F. J. A. M. Houten van and H. J. J. Kals: Journal of Manufacturing Systems Vol. 12 (1993), p. 113-132 